\title{
Mucins, trefoil factors and pancreatic duodenal homeobox 1 expression in spasmolytic polypeptide expressing metaplasia and intestinal metaplasia adjacent to gastric carcinomas
}

\author{
Nuray Can¹, Fulya Oz Puyan¹, Semsi Altaner¹, Filiz Ozyilmaz¹, Burcu Tokuc², Zeynep Pehlivanoglu³, \\ Kemal Ali Kutlu ${ }^{1}$
}

\begin{abstract}
1Department of Pathology, Trakya University Medical Faculty, Edirne, Turkey 2Department of Public Health, Trakya University Medical Faculty, Edirne, Turkey ${ }^{3}$ Department of Pathology, Arnavutkoy State Hospital, Istanbul, Turkey
\end{abstract}

Submitted: 26 September 2011 Accepted: 12 October 2011

Arch Med Sci 2020; 16 (6): 1402-1410

DOI: 10.5114 /aoms.2013.36923

Copyright (c) 2013 Termedia \& Banach

\section{Abstract}

Introduction: Gastric cancers are the second cause of cancer related deaths all around the world but gastric carcinogenesis remains a mystery. Intestinal metaplasia (IM) and spasmolytic polypeptide expressing metaplasia (SPEM) are the two types of preneoplastic metaplasias. In this study, we aimed to investigate expression of Pancreatic duodenal homeobox 1 (PDX1), mucins (MUCS), trefoil factors (TFFs) in SPEM and IM surrounding gastric carcinomas. Material and methods: Tissue samples of tumor adjacent gastric mucosa including IM $(n=61)$ and SPEM $(n=36)$ from 70 gastrectomy specimens were used for immunohistochemical analysis of PDX1, mucins (MUC5AC, MUC6) and trefoil factors (TFF2, TFF3).

Results: Nuclear expression of PDX1 was present in both SPEM (32/36) and IM (60/61) and there was no significant difference in expression of PDX1 between the two types of metaplasias. While TFF3 and MUC5AC were abundant in IM, SPEM showed $100 \%$ expression of TFF2 and MUC6 and also lower positivity with TFF3 and MUC5AC. PDX1 positivity was related to expression of MUC5AC $(60 / 61, p<0.001)$ and TFF3 $(60 / 61, p<0.001)$ in IM and also associated with expression of MUC5AC $(14 / 32, p<0.05)$, MUC6 $(32 / 32, p<0.001)$, TFF2 $(32 / 32, p<0.001)$ and TFF3 $(9 / 32, p<0.05)$ in SPEM. Coexpression of TFF3 and TFF2 was present in 10 of 36 (27.7\%) samples of SPEM and also 29 of 61 (47.5\%) samples of IM exhibited dual expression of trefoil peptides. Conclusions: PDX1 may affect the development of SPEM and IM. Expression patterns of TFFs and MUCS may indicate that IM evolves from SPEM.

Key words: spasmolytic polypeptide expressing metaplasia, intestinal metaplasia, trefoil factors, pancreatic duodenal homeobox 1 , mucins.

\section{Introduction}

Gastric carcinomas are the second cause of cancer related deaths all around the world. Five-year survival rates of these tumors are lower than $20 \%$ but according to the reports in recent years, the 10-year survival rate reaches up to $90 \%$ with early diagnosis and surgery, which is the most effective procedure of therapy [1]. Despite the significant effect of early detection and resection, most patients present with advanced tu-

\author{
Corresponding author: \\ Nuray Can MD, PhD \\ Department of Pathology \\ Trakya University \\ Medical Faculty \\ Edirne 22030, Turkey \\ Phone: +90 41438060 00, \\ +90543 3858197 \\ E-mail: nuraycan@ymail.com
}


mor stage and lose the chance of surgery [1, 2]. The knowledge about the cellular mechanisms of neoplastic progression of preneoplastic lesions to gastric cancer is not clear enough yet. Due to that, if the knowledge of preneoplastic events becomes more comprehensive, gastric cancer mortality may be reduced depending on the discovery of better screening programs for populations at risk [2].

The gastric mucosa exhibits different histological and functional features according to anatomic regions. The cell lineage belonging to normal fundic mucosa bears luminal secretion of mucins (MUCs), intrinsic factor, acid and pepsinogen [3]. In humans, oxyntic atrophy, characterized by the loss of parietal cells, is a prior stage of development of intestinal-type gastric adenocarcinoma [4]. Active chronic gastritis that usually evolves from Helicobacter pylori infection leads to multifocal atrophic gastritis and then metaplastic lineages appear. So, loss of parietal cells seems to be the most important mucosal alteration related to gastric preneoplasia $[5,6]$.

Intestinal metaplasia (IM) and spasmolytic polypeptide (SP)/Trefoil factor peptide 2 (TFF2) expressing metaplasia, namely pseudopyloric metaplasia, also are the two types of metaplasias linked with gastric cancer [6]. Intestinal metaplasia has become a point of interest after it has been accepted as the most prominent pioneer of gastric cancer [7, 8]. In the stomach, presence of cells exhibiting small intestine or large intestine phenotype defines IM [5]. Spasmolytic polypeptide expressing metaplasia (SPEM), which is characterized by the existence of SP expressing mucous cells resembling deep antral gland cells or Brunner's gland cells in the base of the gastric fundus revealing oxyntic atrophy [9] was first recognized in the vicinity of benign gastric ulcers in 1959 [10]. Although there is no direct proof about the link between a specific type of metaplasia and neoplastic progress, in the last decade, research in mice, Mongolian gerbils and humans revealed that SPEM is associated with the evolution of gastric cancer, like IM $[7,8,11,12]$. The most recent report about the relationship between SPEM and IM revealed that IM and also invasive neoplastic lesions evolve from SPEM in amphiregulin-deficient mice [6]. Thus, the relationship between SPEM and IM in humans is becoming a subject of interest.

Current data propose that homeobox proteins can be positive or negative tumor modulators [13] and caudal homeobox gene CDX2 that belongs to ParaHox family transcription factors causes IM in the gastric mucosa [14]. Pancreatic-duodenal homeobox 1 (PDX1), also named as insulin promoter factor 1 (IPF-1), somatostatin transcription factor 1 (STF-1) or Islet/duodenum homeobox 1 (IDX-1), is a transcription factor of the ParaHox gene family $[15,16]$ and it has a crucial role in the development of pancreas, duodenum and gastric antrum [17]. In humans, PDX1 is located at chromosome 13q12.1 adjacent to CDX2 [15]. In recent years, some authors have reported that PDX1 has been expressed in SPEM and IM and also its expression has been associated with MUC6 [18].

Trefoil factor peptides (TFFs) are a family of peptides comprising small secretory peptides bearing one or more trefoil domain [19]. In humans, three kinds of trefoil peptide have been defined: TFF1/pS2 (breast cancer estrogen inducible peptide), TFF2/ Hsp (spasmolytic polypeptide) and TFF3/ITF (intestinal trefoil factor). Principally, mucin secreting cells lining the gastrointestinal tract synthesize and secret TFFs, so that these peptides are closely associated with MUCs. In normal gastric mucosa, TFF1 coordinates with MUC5AC in the surface/foveolar epithelium. TFF2 is expressed together with MUC6 in mucous cells of the regenerating zone of the gastric body, antral glands and also pseudopyloric glands [20]. The last defined trefoil factor, TFF3, correlates with MUC2 and it is mainly expressed in the goblet cells of the small/ large intestine and gastric IM [19, 21].

Mucins are heavily glycosylated glycoproteins which are the major constituents of the mucous viscous gel coating the surface of epithelial cells. Recently, 20 mucin genes have been identified, but in practice, only a few (MUC1, MUC2, MUC5AC and MUC6) are in routine use and even those are used mainly in research settings [22]. Studies showed that all these mucins are distinctly expressed in epithelia with cell type specificity. MUC1, MUC5AC and MUC6 are gastric mucins and the first two of them are found in foveolar/surface epithelium [23]. Since MUC6 is secreted in the regenerative zone of corpus mucosa and in the deep antral glands, it is also present in SPEM [20]. Normally MUC2 is not expressed in gastric mucosa; it is a sign of IM [23]. Although MUC5AC and MUC6 are gastric mucins, they are also markers of incomplete type IM $[1,18]$. Recent knowledge shows that gastric precancerous lesions and neoplasms exhibit altered expression patterns of mucins and TFFs and these alterations may be used as a pointer of tumor progression [20].

In this study, to clarify whether there is a sequential progression from SPEM to IM in humans as mentioned in models of mice and Mongolian gerbils and also PDX1 status in the two types of metaplasias, we investigated the expression of MUC5AC, MUC6, TFF2, TFF3 and PDX1 in IM and SPEM adjacent to gastric cancers.

\section{Material and methods}

\section{Ethics}

This work has been carried out in accordance with the Declaration of Helsinki (2000) of the World Medical Association. This project was ap- 
proved ethically by the ethics committee of Trakya University Medical Faculty (TUTFEK 2008/65).

\section{Tissue samples and histological examination}

In this study, 70 total/partial gastrectomy specimens which were resected between 2006 and 2009 in the Medical Faculty of Trakya University because of primary gastric carcinoma were re-evaluated. Tissue samples of gastric mucosa adjacent tumor containing IM $(n=61)$ and SPEM $(n=36)$ were selected for immunohistochemical studies. The group comprised 54 men and 16 women, aged $26-80$ years (mean \pm standard derivation, $64.62 \pm 11.3)$.

Sections ( $4 \mu \mathrm{m}$ thick) of the paraffin-embedded tissue were stained with hematoxylin and eosin $(H+E)$, and also with periodic-acid Schiff/Alcian blue (PAS/AB) for detecting IM [24]. Histologically, SPEM was defined when antral glands or Brunner's gland like glands were observed in deep corpus mucosa on the $\mathrm{H}+\mathrm{E}$ stained slides.

\section{Immunohistochemical analysis}

For immunohistochemistry, we used polyclonal rabbit antibody for PDX1 (Gene Tex/GTX19379), polyclonal mouse antibody for TFF2 (Abnova/ H00007032-B01P) and monoclonal mouse antibodies for MUC5AC (Gene Tex/GTX18097), MUC6 (Thermo-Scientific/MS -1153-R7) and TFF3 (Ab$\mathrm{cam} / \mathrm{ab} 57752$ ). Sections were kept at $37^{\circ} \mathrm{C}$ overnight and continued deparaffinizing in xylene, rehydrated in ethanol and then treated with $0.3 \%$ hydrogen peroxide in methanol for $10 \mathrm{~min}$ at $37^{\circ} \mathrm{C}$ to inhibit endogenous peroxidase activity. The slides were put in phosphate buffered saline (PBS), pH 7.4 for $10 \mathrm{~min}$ and instilled with Large Volume Ultra $\vee$ Block (Code No: TA-125-UB, Neomarkers Fremont, CA, US). Subsequently, sections were incubated overnight at $4^{\circ} \mathrm{C}$ with anti-PDX1, anti-TFF2, anti-MUC5AC, anti-MUC6 and antiTFF3. After washing with distilled water, slides were treated with PBS for 5 min. Sections were treated with Ultravision Large Volume Detection System Anti-Polyvalent, HRP (Code No: TA 125-HL, Neomarkers, Fremont, CA, US) and Biotinylated Goat Anti-Polyvalent (Code No: TP-125-BN, Neomarkers, Fremont, CA, US) for $1 \mathrm{~h}$, washed with PBS for 5 min, incubated with 'Streptavidin Peroxidase' (Code No: TS-125-HR, Neomarkers, Fremont, CA, US) for $1 \mathrm{~h}$ and then with Ultravision Detection System Large Volume AEC Substrate System (RTU) (Code No: TA-125-HA, Neomarkers, Fremont, CA, US) for $30 \mathrm{~min}$. All specimens were counterstained with Mayer's hematoxylin.

While normal antral mucosa was used as a positive control for MUC5AC, MUC6 and TFF2, normal duodenal mucosa and normal pancreatic tissue were used for TFF3 and PDX1, respectively. Cyto- plasmic staining in more than $10 \%$ of the sample was accepted as positive staining for MUC5AC, MUC6, TFF2 and TFF3. Although we evaluated both cytoplasmic and nuclear staining of PDX1 for gastric cancers in our previous report [25], in this study only nuclear staining was regarded as evaluable for PDX1. According to Leys et al. [26] a semiquantitative scoring system relying on the intensity and extent of staining ( 0 : negative, $1+$, $2+, 3+)$ was used; scores $1+, 2+, 3+$ were rendered as positive.

\section{Statistical analysis}

Statistical analyses were performed using Statistica 7.0 (StatSoft Inc. Tulsa, OK, USA) statistical software. The $\chi^{2}$ test or Fisher exact tests were used to analyze the significance of difference or relations between variables. Values of $p \leq 0.05$ was considered statistically significant.

\section{Results}

Among the 70 cases, 16 (22.8\%) were female and 54 (77.2\%) were male. Sixty-one samples of IM were selected by histological examination of $\mathrm{H}+\mathrm{E}$ slides and then confirmed by observing $A B$ positivity in goblet cells by $P A S / A B$ staining. In $\mathrm{H}+\mathrm{E}$ slides, 36 samples of cancer adjacent corpus mucosa contained antral glands or Brunner's gland like mucous glands which are defined as SPEM. We determined SPEM surrounding IM especially close to lymphoid follicles in atrophic corpus mucosa in 32 of 36 (88.9\%) samples of SPEM. In some of the samples containing the two types of metaplasias, intervening glands resembling both SPEM and IM were conspicuous.

\section{Immunohistochemical analysis of PDX1, MUC5AC, MUC6, TFF2 and TFF3 expression in $I M$}

We found nuclear and cytoplasmic PDX1 expression in $\mathrm{IM}$, but we regarded nuclear staining as positivity in this study. Intensive PDX1 positivity was determined in the nuclei of goblet cells in nearly all samples (60/61, 98.3\%). Cytoplasmic expression of TFF3 in goblet cells was present in all samples (61/61, 100\%), and expression was strong and diffuse. Cytoplasmic positivity of TFF2, MUC6 and MUC5AC was detected in 29 (47.5\%), 2 (3.2\%) and $60(98.3 \%)$ of 61 samples of IM, respectively (Table I, Figure 1). We evaluated that especially goblet cells of IM localized in the superficial mucosa exhibited higher positivity of MUC5AC than in the deeper glands (Figure 1). PDX1 positivity was related to expression of MUC5AC and TFF3 in IM (60/61, $p<0.001 ; 60 / 61, p<0.001$, respectively). However, there was no correlation between PDX1 positivity and MUC6 or TFF2 expressions in IM (Table II). 
Immunohistochemical analysis of PDX1, MUC5AC, MUC6, TFF2 and TFF3 expression in SPEM

Nuclear PDX1 expression in SPEM was present in 32 of 36 (88.9\%) samples (Table I). Although we aimed to analyze the nuclear positivity of PDX1 in mucous cells of SPEM, cytoplasmic positivity was also noted. Cytoplasmic positivity of TFF2 and MUC6 was present in all samples (36/36, 100\%), as expected. MUC6 positivity was strong and diffuse, but expression of TFF2 was variable. TFF3 and MUC5AC positivity was detected in $10(27.7 \%)$ and 14 (38.8\%) of 36 samples of SPEM, respectively (Table I, Figure 2). We found that PDX1 positivity was related to expression of MUC5AC $(14 / 32, p<0.05)$, MUC6 (32/32, $p<0.001)$, TFF2 (32/32, $p<0.001)$ and TFF3 $(9 / 32, p<0.05)$ in SPEM (Table II).

Comparison of PDX1, MUC5AC, MUC6, TFF2 and TFF3 expression in SPEM and IM

There was no significant difference between the expression of PDX1, MUC5AC, MUC6, TFF2 and TFF3 in SPEM and IM (Table I, Figure 3). However, some differences which are not significant occurred. PDX1 reactions were not strong and
Table I. Comparison of pancreatic duodenal homeobox-1 (PDX1), mucins (MUC5AC, MUC6) and trefoil factors (TFF2, TFF3) expression in intestinal metaplasia (IM) and spasmolytic polypeptide expressing metaplasia (SPEM)

\begin{tabular}{|lccc|}
\hline Antibodies & $\begin{array}{c}\text { IM }(N=61) \\
n(\%)\end{array}$ & $\begin{array}{c}\text { SPEM }(N=36) \\
n(\%)\end{array}$ & $P$-value \\
\hline PDX1 (+) & $60(98.3)$ & $32(88.9)$ & $1.000^{*}$ \\
\hline MUC5AC (+) & $60(98.3)$ & $14(38.9)$ & $1.000^{*}$ \\
\hline MUC6 (+) & $2(3.3)$ & $36(100.0)$ & $1.000^{\dagger}$ \\
\hline TFF2 (+) & $29(47.5)$ & $36(100.0)$ & $0.8^{*}$ \\
\hline TFF3 (+) & $61(100.0)$ & $10(41.7)$ & $0.4^{*}$ \\
\hline
\end{tabular}

${ }^{*} \chi^{2}$ test, Fisher's exact test.

diffuse in SPEM as in IM (Figure 4). We recognized that TFF3 positivity was strong and diffuse in $I M$ (Figures 1 and 5), while the expression of MUC5AC was weak, focal and localized especially superficially (Figure 1). TFF2 and MUC6 reactivity overlapped with mucous glands defined as SPEM by $\mathrm{H}+\mathrm{E}$ staining (Figures 2 and 5). Coexpression of TFF3 and TFF2 was present in 10 of $36(27 \%)$ samples of SPEM and also 29 of $61(47.5 \%)$ samples of IM exhibiting dual expression of trefoil peptides (Figure 5).
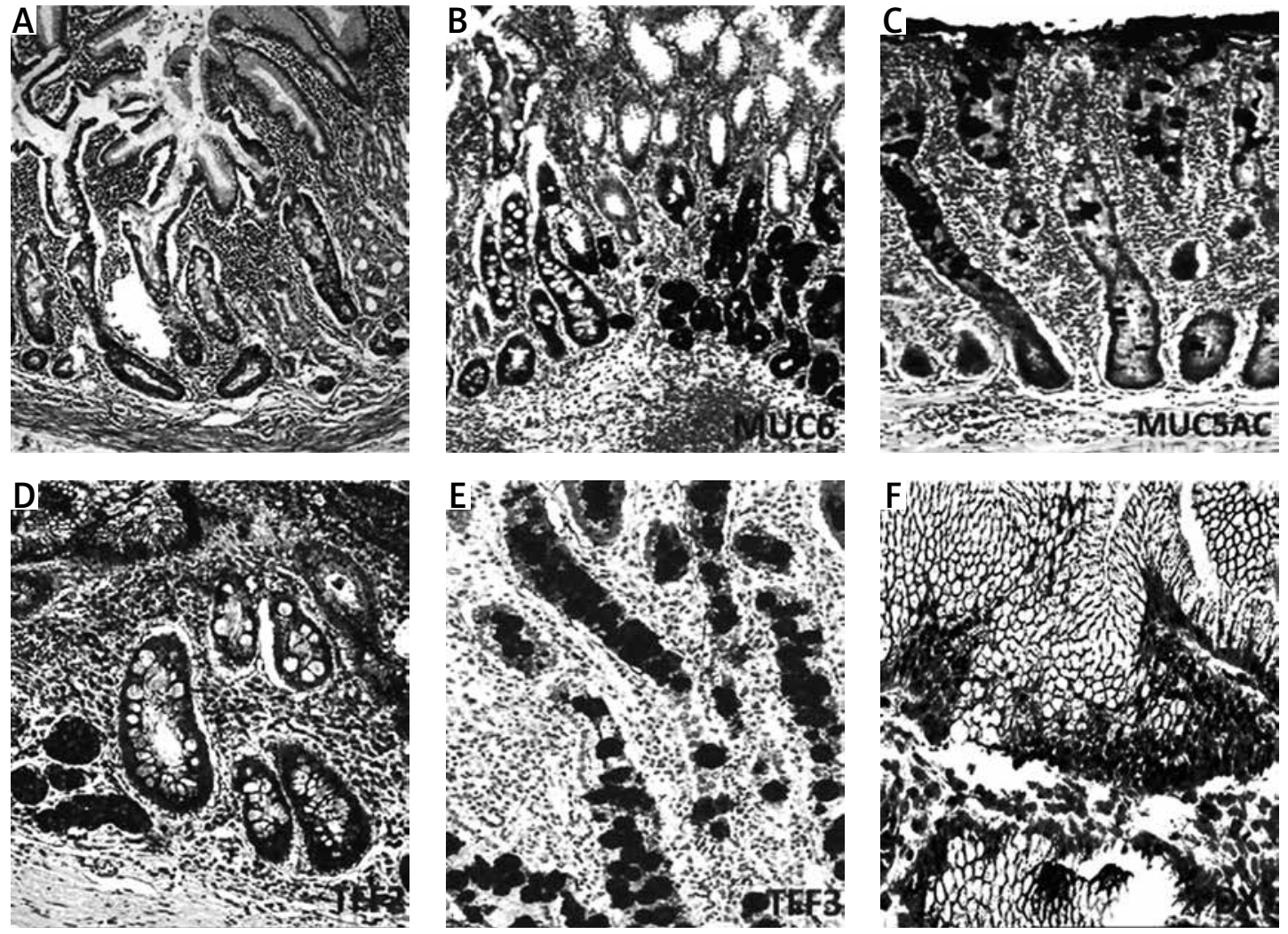

Figure 1. Mucins (MUCs), trefoil factors (TFFs) and pancreatic duodenal homeobox 1 (PDX1) expression in intestinal metaplasia (IM). A - Histological view of IM (H + E stain 50x). B - Weak expression of MUC6 in IM (IHC 100x). C - MUC5AC positivity in IM. Especially superficial localization of the positivity should be noted (IHC 100x). D-Weak positivity of TFF2 in IM (IHC 200x). E-Strong and diffuse cytoplasmic positivity with TFF3 in goblet cells of IM (IHC 100x). F - Diffuse nuclear immunopositivity of PDX1 in IM (IHC 200x) 
Table II. Relationship of pancreatic duodenal homeobox-1 (PDX1) expression with mucins (MUC5AC, MUC6) and trefoil factors (TFF2, TFF3) in intestinal metaplasia (IM) and spasmolytic polypeptide expressing metaplasia (SPEM)

\begin{tabular}{|lcccc|}
\hline Types of metaplasia/PDX1 & MUC5AC $(+)$ & MUC6 $(+)$ & TFF2 $(+)$ & TFF3 (+) \\
\hline IM $(n=61):$ & & & & \\
\hline PDX1 $(+)(n=60)$ & 60 & 2 & 0 & 60 \\
\hline PDX1 $(-)(n=1)$ & 1 & 0 & 0.08 & 1 \\
\hline$P$-value & $0.000^{\text {a }}$ & 1.000 & 32 & $0.000^{\text {a }}$ \\
\hline SPEM $(n=36):$ & & & 4 & 9 \\
\hline PDX1 $(+)(n=32)$ & 14 & 32 & $0.000^{\text {a }}$ & 1 \\
\hline PDX1 $(-)(n=4)$ & 0 & 4 & $0.04^{\text {b }}$ \\
\hline$P$-value & $0.007^{\text {b }}$ & $0.00^{\text {a }}$ & & \\
\hline
\end{tabular}

${ }^{\star}$ Fisher's exact test, ${ }^{a} p<0.001,{ }^{b} p<0.05$
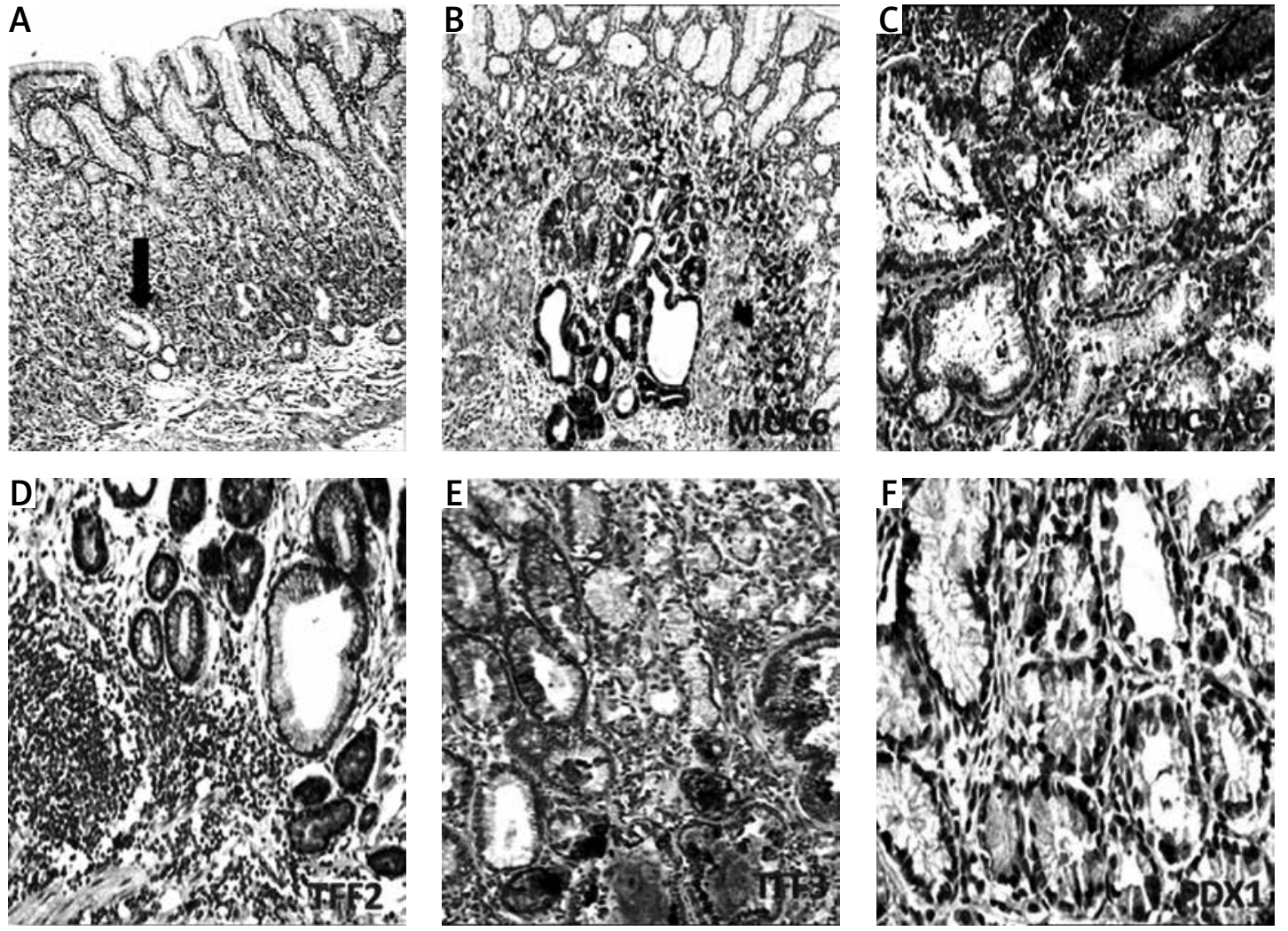

Figure 2. Mucins (MUCs), trefoil factors (TFFs) and pancreatic duodenal homeobox 1 (PDX1) expression in spasmolytic polypeptide expressing metaplasia (SPEM). A - SPEM in basal portion of corpus mucosa (arrow) $(\mathrm{H}+\mathrm{E}$ stain 50x). B - Diffuse and strong expression of MUC6 in SPEM. Note the staining pattern of the normal corpus mucosa surrounding pseudopyloric glands (IHC 100x). C - SPEM exhibiting weak and focal MUC5AC positivity (IHC 200x). D - Strong and diffuse positivity of TFF2 in SPEM (IHC 200x). E-Cytoplasmic positivity with TFF3 in some of the metaplastic pseudopyloric glands. Note the staining pattern in the form of droplets in some mucous cells (IHC 200x). F - Weak nuclear immunopositivity of PDX1 in SPEM (IHC 400x)

\section{Discussion}

After Correa [7] had described the sequence of distinct steps including metaplasia in carcinogenesis of intestinal type gastric cancer, the role of IM as a putative precursor to gastric neoplasia in the scenario of metaplasia to cancer became the subject of investigations. Current studies show that another type of gastric metaplasia, SPEM, is also related to gastric cancers. However, the final relationships between these two types of metaplasias and gastric cancer have not been clarified yet [6]. In recent reports, it is believed that the homeobox gene PDX1 can be a part of gastric carcinogenesis $[27,28]$ and also it can affect the phenotype of the occurring gastric cancer, as we reported in our previous paper [25]. To investigate the role of PDX1 in IM and SPEM which are preneoplastic and 
the relation between these two types of metaplasias according to expression of mucins and trefoil peptides, we examined PDX1 reactivity and also the expression of trefoil peptides (namely, TFF2 and TFF3) and mucins (MUC5AC and MUC6) by immunohistochemistry in tumor surrounding mucosa. SPEM was associated with up to $90 \%$ of resected gastric cancers in three different studies [29-31]. A recent report informed that $I M$ and invasive fundic dysplastic lesions evolve from SPEM in amphiregulin-deficient mice [6]. Finally, Goldenring et al. [32] reported compound glands containing SPEM cells in the deep portions and intestinal metaplastic lineages in the luminal portions adjacent to gastric cancer in human resection materials. In the present study, we also observed that SPEM is also associated with gastric cancer as IM and coexistence of IM and SPEM in a nested localization of human gastric mucosa adjacent cancers.

Mucins are distinctly expressed in epithelia with cell type specificity [23]. MUC5AC is a type of mucin expressed in the gastric foveolar epithelium and its expression is expected more often in incomplete type of $\mathrm{IM}$ rather than complete type. According to present data, incomplete type IM associates with dysplasia or cancer more often than complete type [33]. Although the aim of analyzing MUC5AC expression in this study was to isolate the foveolar epithelium from deep glands and not

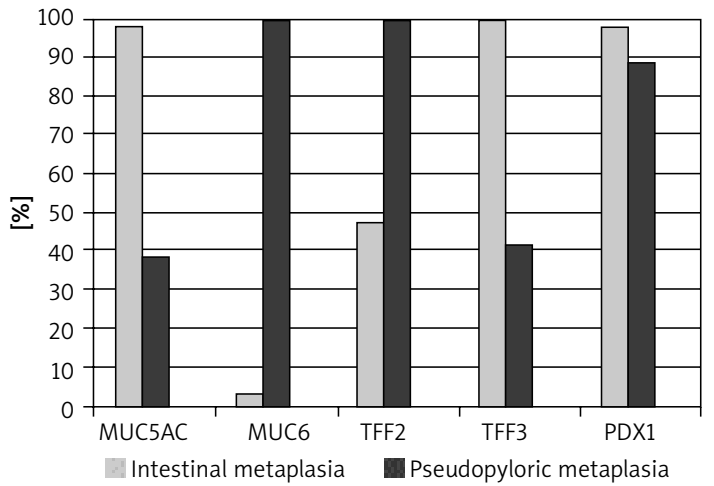

Figure 3. Mucins (MUC5AC, MUC6), trefoil factors (TFF2, TFF3) and pancreatic duodenal homeobox 1 (PDX1) expression in spasmolytic polypeptide expressing metaplasia (SPEM) and intestinal metaplasia (IM)

to classify subtypes of IM, we observed MUC5AC expression with variable intensity in nearly all of the samples of IM. We also recognized that TFF3 positivity accompanied MUC5AC positivity in IM. According to these findings, expression of $\mathrm{MU}$ C5AC in IM clarified that our samples included incomplete type $I M$. This finding points out the relation of MUC5AC with incomplete type IM [5]. According to this result, incomplete type IM can be accepted to be a more important suspect of gastric cancer than complete type, relying on the idea which is supported by Dinis-Ribeiro et al.'s study [34].
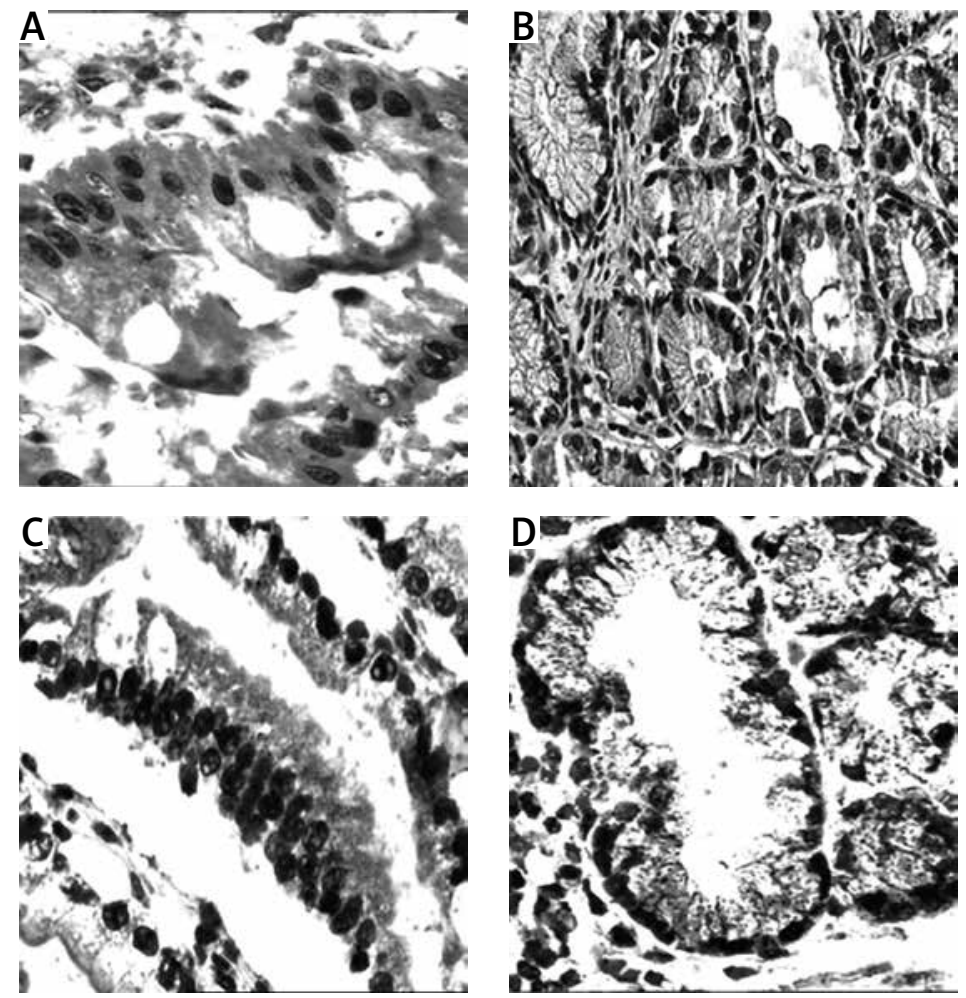

Figure 4. Pancreatic duodenal homeobox 1 (PDX1) expression in spasmolytic polypeptide expressing metaplasia (SPEM) and intestinal metaplasia (IM). A, B - Strong PDX1 expression in the nuclei of IM (IHC 400x). C, D - Weak nuclear expression of PDX1 in SPEM (IHC 400x) 

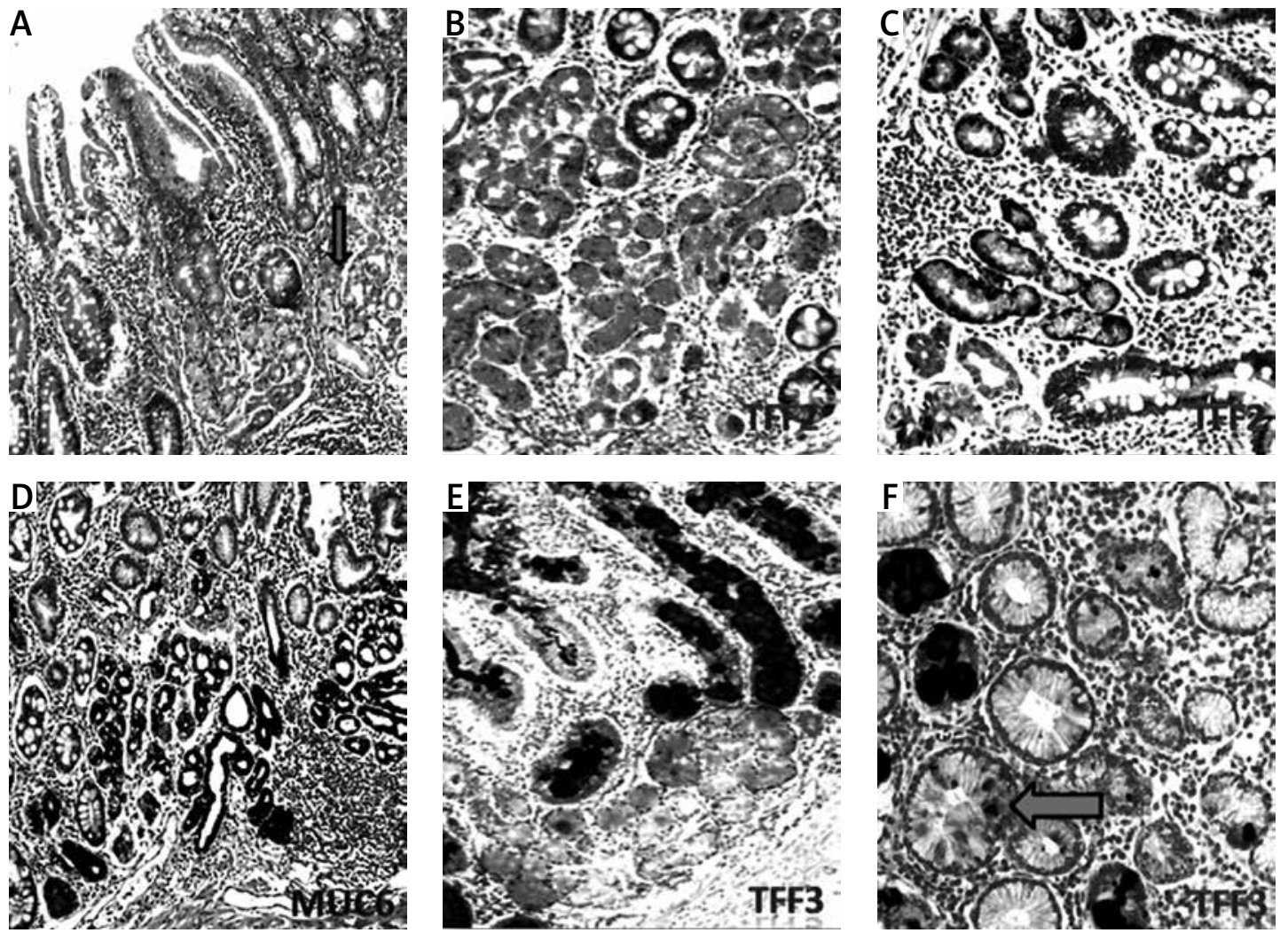

Figure 5. Mucin (MUC6) and trefoil factors (TFF2, TFF3) expression in the interface regions between spasmolytic polypeptide expressing metaplasia (SPEM) and intestinal metaplasia (IM). A - Corpus mucosa exhibiting IM nested with SPEM (arrow) (H + E stain 50x). B, C - TFF2 staining; strong positivity in SPEM and weak expression in IM (IHC 200x). D - Strong expression of MUC6 in SPEM and in some of the glands in the interface region between IM and SPEM (IHC 100x). E - Strong and diffuse cytoplasmic positivity with TFF3 in goblet cells of IM and in some of the metaplastic pseudopyloric glands. Note the increasing intensity of the staining in SPEM localized adjacent to IM (IHC 100x). F-The expression of TFF3 in the form of droplets in some of the mucous cells of SPEM (arrow) (IHC 400x)

In agreement with previous reported data, IM strongly expresses PDX1, especially in the nuclei of goblet cells $[17,26]$, but a controversy still continues about PDX1 expression in SPEM. Sakai et al. [17] reported that PDX1 plays an important role in the development of SPEM. According to the results of Leys et al. [26], SPEM did not exhibit nuclear PDX1 positivity. We demonstrated the presence of weaker nuclear PDX1 expression in SPEM than IM and its expression had a significant relation with the expressions of TFF2, TFF3, MUC6 and MUC5AC in SPEM and also with positivity of TFF3 and MUC5AC in IM. The correlation between PDX1 and TFF2, TFF3, MUC5AC and MUC6 may be a result of the role of the gene which is taking part in the development of the gastric antrum and duodenum. Although PDX1 is expressed in both IM and SPEM, the intensity of expression is variable in IM. This finding may be due to the different cell components and different contents of mucins in the small and large intestine $[8,18]$. This problem can be solved by a study design comparing PDX1 expression in IM and subtypes of IM by demonstrating specific mucins such as MUC5AC, MUC6, MUC2 and cell types indicating the small or large intestine.
We recognized that IM surrounded most of the foci of SPEM and we also noticed the presence of glands consisting of two types of gastric metaplastic lineages. Ten of 36 samples of SPEM expressed TFF3 in the cytoplasm of mucous cells, and also nine of these ten samples showed nuclear PDX1 positivity that correlated with TFF3 expression significantly. Dual TFF2 and TFF3 positivity was seen in nearly half of the foci of intestinal metaplasia and in one third of SPEM samples. Nam et al. [6] reported dual expression of TFF2 and MUC2 or TFF2 and TFF3 in the interface regions of IM and SPEM in mice. As the meaning of transformation of SPEM to IM, dual staining of MUC2 and TFF2 in SPEM glands was demonstrated by Yoshizawa et al. [12] in Mongolian gerbils and also presented by Goldenring et al. [32] in humans, previously. These findings suggest that SPEM and IM can represent sequential metaplastic steps concordant with recent data of mice models [6] and the cells expressing PDX1, and that TFF3 in the foci of SPEM may be transforming cells from mucous cells to goblet cells. Wright et al. [35, 36] have reported this kind of metaplastic conversion in the ulcer-associated cell lineage in Crohn's disease. When the 
results of the present study in humans are taken together with the previous results of studies in mice [6], Mongolian gerbils [12], and recently, in humans [32], the metaplastic process may start with the emergence of SPEM in the setting of oxyntic atrophy and go on by evolution of IM.

In conclusion, our results suggest that PDX1 may have an effect on the development of SPEM and $I M$, and trefoil factors and mucins can be useful indicators to determine the type of developing gastric metaplastic processes. The unexpected expression of TFF3 in SPEM and dual expressions of TFF2 and TFF3 in IM and SPEM may indicate that IM may evolve from SPEM, which can be a step between atrophy and $I M$ in the gastric carcinogenesis schema conceived by Corea [7]. Pathologists and gastroenterologists should be aware of the message of pseudopyloric glands emerging in atrophic corpus mucosa, because they can be a messenger of forthcoming IM or cancer.

\section{Acknowledgments}

This work was supported by Trakya University Scientific Research Projects (TUBAP), No. 110/08.

\section{Conflict of interest}

The authors declare no conflict of interest.

\section{References}

1. Lauwers GY. Epithelial neoplasms of the stomach. In: Surgical pathology of the Gl tract, liver, biliary tract and pancreas. Odze RD, Goldblum JR (eds). Saunders Elsevier, Philadelphia 2009; 563-81.

2. Weis VG, Goldenring JR. Current understanding of SPEM and its standing in the preneoplastic process. Gastric Cancer 2009; 12: 189-97.

3. Karam SM, Leblond CP. Dynamics of epithelial cells in the corpus of the mouse stomach. I. Identification of proliferative cell types and pinpointing of the stem cell. Anat Rec 1993; 236: 259-79.

4. El-Zimaity HM, Ota H, Graham DY, Akamatsu T, Katsuyama T. Patterns of gastric atrophy in intestinal type gastric carcinoma. Cancer 2002; 94: 1428-36.

5. Cecilia M, Fenoglio-Preiser C, Noffsinger A, Stemmermann GN, Lantz PE, Isaacson PG. The nonneoplastic stomach. In: Gastrointestinal pathology: an atlas and text. Fenoglio-Preiser C (ed.). Lippincott Williams \&Wilkins, Virginia 2008; 200-33.

6. Nam KT, Lee HJ, Mok H, et al. Amphiregulin-deficient mice develop spasmolytic polypeptide expressing metaplasia and intestinal metaplasia. Gastroenterology 2009; 136: 1288-96.

7. Correa P. Human gastric carcinogenesis: a multistep and multifactorial process. First American Cancer Society Award Lecture on Cancer Epidemiology and Prevention. Cancer Res 1992; 52: 6735-40.

8. Filipe MI, Munoz N, Matko I, et al. Intestinal metaplasia types and the risk of gastric cancer: a cohort study in Slovenia. Int J Cancer 1994; 57: 324-9.

9. Goldenring JR, Nomura S. Differentiation of the gastric mucosa III. Animal models of oxyntic atrophy and meta- plasia. Am J Physiol Gastointest Liver Physiol 2006; 291: 999-1004.

10. El-Zimaity HM. Gastric atrophy, diagnosing and staging. World J Gastroenterology 2006; 36: 5757-62.

11. Correa P, Houghton J. Carcinogenesis of Helicobacter pylori. Gastroenterology 2007; 133: 659-72.

12. Yoshizawa N, Takenaka Y, Yamaguchi Y, et al. Emergence of spasmolytic polypeptide-expressing metaplasia in Mongolian gerbils infected with Helicobacter pylori. Lab Invest 2007; 87: 1265-76.

13. Abate-Shen C. Deregulated homeobox gene expression in cancer: cause or consequence? Nat Rev Cancer 2002; 2: 777-85.

14. Silberg DG, Sullivan J, Kang E, et al. Cdx2 ectopic expression induces gastric intestinal metaplasia in transgenic mice. Gastroenterology 2002; 122: 689-96.

15. Brooke NM, Garcia-Fernandez J, Holland PW. The ParaHox gene cluster is an evolutionary sister of the Hox gene cluster. Nature 1998; 392: 920-2.

16. Ohlsson H, Karlsson K, Edlund T. IPF1, a homeodomain-containing transactivator of the insulin gene. EMBO J 1993; 12: 4251-9.

17. Sakai H, Eishi $Y$, Li XL, et al. PDX1 homeobox protein expression in pseudopyloric glands and gastric carcinomas. Gut 2004; 53: 323-30.

18. Tsukamoto T, Mizoshita T, Tatematsu M. Gastric-and-intestinal mixed-type intestinal metaplasia: aberrant expression of transcription factors and stem cell intestinalization. Gastric Cancer 2006; 9: 156-66.

19. Leung WK, Yu J, Chan FK, et al. Expression of trefoil peptides (TFF1, TFF2, and TFF3) in gastric carcinomas, intestinal metaplasia, and non-neoplastic gastric tissues. J Pathol 2002; 197: 582-8.

20. Xia HX, Yang Y, Lam SK, et al. Aberrant epithelial expression of trefoil family factor 2 and mucin 6 in Helicobacter pylori infected gastric antrum, incisura, and body and its association with antralisation. Clin Pathol 2004; 57: 861-6.

21. Kang W, Rathinavelu S, Samuelson LC, Merchant JL. Interferon gamma induction of gastric mucous neck cell hypertrophy. Lab Invest 2005; 85: 702-15.

22. Bu XD, Li N, Tian XQ, et al. Altered expression of MUC2 and MUC5AC in progression of colorectal carcinoma. World J Gastroenterol 2010; 16: 4089-94.

23. Babu SD, Jayanthi V, Devaraj N, Reis CA, Devaraj H. Expression profile of mucins (MUC2, MUC5AC and MUC6) in Helicobacter pylori infected pre-neoplastic and neoplastic human gastric epithelium. Mol Cancer 2006; 5: 10.

24. Goldenring JR, Ray GS, Coffey RJ, et al. Reversible drug-induced oxyntic atrophy in rats. Gastroenterology 2000; 118: 1080-93.

25. Oz Puyan F, Can N, Ozyilmaz F, et al. The relationship among PDX1, CDX2, and mucin profiles in gastric carcinomas: correlations with clinicopathologic parameters. J Cancer Res Clin Oncol 2011; 137: 1749-62.

26. Leys CM, Nomura S, Rudzinski E, et al. Expression of $\mathrm{Pdx}-1$ in human gastric metaplasia and gastric adenocarcinoma. Hum Pathol 2006; 37: 1162-8.

27. Busuttil RA, Boussioutas A. Intestinal metaplasia: a premalignant lesion involved in gastric carcinogenesis. J Gastroenterol Hepatol 2009; 24: 193-201.

28. Wang XP, Li ZJ, Magnusson J, Brunicardi FC. Tissue microarray analyses of pancreatic duodenal homeobox- 1 in human cancers. World J Surg 2005; 29: 334-8.

29. Schmidt PH, Lee JR, Joshi V, et al. Identification of a metaplastic cell lineage associated with human gastric adenocarcinoma. Lab Invest 1999; 79: 639-46. 
30. Halldorsdottir AM, Sigurdardottir M, Jonasson JG, et al. Spasmolytic polypeptide expressing metaplasia (SPEM) associated with gastric cancer in Iceland. Dig Dis Sci 2003; 48: 431-41.

31. Yamaguchl H, GoldenrIng JR, KamInIshI M, Lee JR. Identification of spasmolytic polypeptide expressing metaplasia (SPEM) in remnant gastric cancer and surveillance postgastrectomy biopsies. Dig Dis Sci 2002; 47: 573-8.

32. Goldenring JR, Nam KT, Wang TC, Mills JC, Wright NA. Spasmolytic polypeptide-expressing metaplasia and intestinal metaplasia: time for reevaluation of metaplasias and the origins of gastric cancer. Gastroenterology 2010; 138: 2207-10.

33. Cecilia M, Fenoglio-Preiser C, Noffsinger A, Stemmermann GN, Lantz PE, Isaacson PG. The neoplastic stomach. In: Gastrointestinal pathology: an atlas and text Fenoglio-Preiser C (ed.). Lippincott Williams \&Wilkins, Virginia 2008; 233-75.

34. Dinis-Ribeiro M, Lopes C, Costa Pereira A, et al. A follow up model for patients with atrophic chronic gastritis and intestinal metaplasia. J Clin Pathol 2004; 57: 177-82.

35. Wright NA, Pike C, Elia G. Induction of a novel epidermal growth factor-secreting cell lineage by mucosal ulceration in human gastrointestinal stem cells. Nature 1990; 343: 82-5.

36. Wright NA, Poulsom R, Stamp GW, et al. Epidermal growth factor (EGF/URO) induces expression of regulatory peptides in damaged human gastrointestinal tissues. J Pathol 1990; 162: 279-84. 